\title{
BIENESTAR EMOCIONAL, PSICOLÓGICO Y SOCIAL EN ADULTOS ARGENTINOS EN CONTEXTO DE PANDEMIA POR COVID-19
}

\author{
EMOTIONAL, PSYCHOLOGICAL AND SOCIAL WELL-BEING IN ARGENTINE ADULTS \\ IN THE CONTEXT OF A COVID-19 PANDEMIC
}

\author{
LEANDRO EIDMAN ${ }^{1,2, *}$, VANESA BENDER ${ }^{1}$, JULIETA ARBIZU ${ }^{1,3}$, \\ ADRIANA TAMARA LAMBOGLIA ${ }^{1,4}$, LUCILA CORREA DEL VALLE ${ }^{1,5}$ \\ ${ }^{1}$ RED DE PSICÓlOGOS ESPECIALIZAdOS EN CATÁSTROFES ARGENTINA \\ ${ }^{2}$ UNIVERSIDAD DE CIENCIAS EMPRESARIALES Y SOCIALES \\ ${ }^{3}$ UNIVERSIDAD NACIONAL DE MAR DEL PLATA \\ ${ }^{4}$ UNIVERSIDAD CATÓLICA ARGENTINA \\ ${ }^{5}$ UNIVERSIDAD NACIONAL DE CÓRDOBA
}

FECHA RECEPCIÓN: 24/07/2019・ FECHA ACEPTACIÓN: 1/11/2020

Para citar este artículo: Eidman, L. (2020). Bienestar emocional, psicológico y social en adultos argentinos en contexto de pandemia por COVID-19. Psychologia, 14(2), 69-80 doi: 10.21500/19002386.4851

\section{Resumen}

El objetivo del presente trabajo fue determinar el bienestar emocional, psicológico y social en adultos argentinos en contexto de pandemia por COVID-19. El diseño metodológico utilizado fue no experimental de corte transversal, bajo la modalidad de estudio descriptivo y de diferencia de grupos. Se trabajó con una muestra de 888 participantes, residentes en diferentes provincias de Argentina, de edades comprendidas de entre 18 y 84 años $\left(M_{E D A D}=36.98\right.$; $D E=15.37 ; 51.2 \%$ mujeres, $48.8 \%$ varones). Los resultados informaron una disposición mayoritaria a experimentar niveles de salud mental languideciente. Las personas que se ubicaron en el polo languideciente del continuo de salud mental presentaron menores niveles de bienestar psicológico. El 56.2 \% de los participantes refirió que la mayoría de las veces busca expresar sus emociones con su círculo cercano y el 43 \% informó mantener el contacto con sus seres queridos de forma virtual. Se encontró un mayor nivel de bienestar emocional, psicológico y social en personas que expresaron sus emociones y mantuvieron contacto con sus seres queridos de forma virtual. En conclusión, frente a la situación epidemiológica causada por la pandemia de COVID-19 los adultos argentinos presentaron alteraciones

* https://orcid.org/0000-0002-4553-4773 . leaneidman@gmail.com . Pellegrini 406. Tel: 5493624653979. 
significativas en los niveles de bienestar emocional psicológico y social con predominio de un estado de salud mental languideciente.

Palabras Clave: Bienestar emocional, bienestar psicológico, bienestar social, pandemia por COVID-19

\begin{abstract}
The aim of this study was to determine the emotional, psychological and social well-being in Argentinian adults during the COVID-19 pandemic context. The applied methodological design was cross-sectional not experimental, under descriptive study and group difference modalities. It was used a sample of 888 participants residing in different Argentina provinces, rating ages between 18 and 84 years old $\left(M_{\text {age }}=36.98 ; S D=15.37 ; 51.2 \%\right.$ Female, $48.8 \%$ Male). The results reported a majority susceptibility to experiencing languishing levels of mental health. People who were at the languishing pole of the mental health continuum presented lower levels of psychological well-being. $56.2 \%$ of the participants reported that most of the time they seek to express their emotions within their close circle, and $43 \%$ reported maintaining contact with beloved ones virtually. A higher level of emotional, psychological and social wellbeing was found in people who was able to express their emotions and kept in contact with their beloved ones virtually. In the face of the epidemiological situation caused by the COVID-19 pandemic, Argentinean adults presented significant alterations in the levels of emotional, psychological and social well-being with a predominance of a state of languishing mental health.
\end{abstract}

Keywords: Emotional well-being, psychological well-being, social well-being, COVID-19 pandemic

\section{Introducción}

A fines de diciembre de 2019, un nuevo agente etiológico, denominado SARS-CoV-2, fue identificado como causante de casos de neumonía grave en la ciudad china de Wuhan (Organización Panamericana de la Salud, 2020). En pocas semanas el virus se esparció por todo el mundo produciendo una alta contagiosidad. La mayoría de los países afectados cerraron sus fronteras y trataron de aislar casos, rastrear contactos y proteger a las poblaciones vulnerables, implementando medidas de distanciamiento físico, que se aplicaron con controles que iban desde recomendaciones hasta multas y arrestos (OMS, 2020).

Se podría situar al agente patógeno como el causante de la mayor infección respiratoria de las últimas décadas; no obstante, existieron enfermedades similares previas, tales como el síndrome respiratorio agudo severo (SARS, por sus siglas en inglés) y el síndrome respiratorio del Medio Oriente (MERS, por sus siglas en inglés), las cuales evidencian antecedentes que permiten predecir las consecuencias para la salud en general y la psicología en particular.

\section{Consecuencias psicológicas derivadas de epidemias y pandemias previas al COVID-19}

En el 2003, el mundo se vio afectado por la mortal epidemia del SARS. Dadas las exigencias y demandas de esta enfermedad para la comunidad, Lee et al. (2007) analizaron las consecuencias psicológicas en sobrevivientes, quienes frente al Cuestionario (GHQ12) y la Escala de Estrés Percibido (PSS-10) mostraron que una proporción del $64 \%$, aún mantenían niveles de estrés y niveles de angustia psicológica.

Luego, en el año 2012 el MERS fue identificado como una nueva infección respiratoria en Arabia Saudita (Zaki et al., 2012), y en Corea en el año 2015 impactó por su rápido contagio y muertes. Jeong et al. (2012) estudiaron el efecto del MERS en la salud mental de la población general en cuarentena por haber estado expuesta al virus. Los resultados informaron presencia de sintomatología ansiosa y sentimientos de rabia; concluyeron que estos problemas de salud mental pueden prevenirse al proporcionar apoyo de salud mental. Los resultados informaron presencia de sintomatología ansiosa (7.6\%) y sentimientos de rabia (16.6\%) durante el período del aislamiento; y tras seis meses después de la cuarentena aún se mantenía un $3 \%$ de síntomas ansiosos y un $6.4 \%$ de sentimientos de rabia; 
concluyeron que estos problemas pueden prevenirse al proporcionar apoyo de salud mental.

Es así como hace tiempo se sabe que las epidemias y las pandemias afectan la salud mental, por lo cual los problemas mentales provocados por brotes virales se han descrito como una epidemia paralela (Hale et al., 2020; Yao et al., 2020). Estudios anteriores revelan que las personas bajo encierro son más propensas a desarrollar varios síntomas de problemas psicológicos, por ejemplo, estrés, depresión, fatiga emocional, insomnio y signos de ansiedad (Brooks et al., 2020).

Actualmente, ante la situación de pandemia por COVID-19, una investigación realizada en población belga reveló que solo el $15 \%$ de los ciudadanos de entre 18 y 65 años antes del brote epidémico presentaron sintomatología relacionada a la ansiedad; sin embargo, luego de dos semanas el estrés de la población aumentó al 25 \% (Hoof, 2020). En la misma línea, un estudio realizado por Crisis Text line informó que en el Reino Unido, EE. UU. y Canadá, a partir de febrero de 2020, hasta el $80 \%$ de los mensajes están relacionados con síntomas de ansiedad y depresión (Chen et al., 2020). Además, Wang et al. (2020) evaluaron el impacto psicológico al principio del brote de COVID-19 en China; los resultados mostraron que el $53.8 \%$ de los participantes experimentaron principalmente ansiedad, depresión, soledad, estrés y miedo, emoción o una combinación de todos estos. En detalle, el $16.5 \%$, el $28.8 \%$ y el $8.1 \%$ de los encuestados informaron niveles moderados a severos de depresión, ansiedad y estrés, respectivamente. Los autores concluyen que los miedos relacionados con la infección, la ansiedad generalizada, la frustración, el aburrimiento y la soledad han sido los agentes que perjudicaron el bienestar subjetivo, la calidad de vida y la capacidad de recuperación.

Pasando ahora al ámbito local, la pandemia por COVID-19 ha generado una mayor demanda de atención psicológica de la población, al presentar elevados porcentajes de síntomas de ansiedad como, por ejemplo, insomnio, preocupación, angustia, distractibilidad, bajo apetito y cansancio (Moreno-Proaño, 2020). En la misma línea, Guiroy et al. (2020) informaron que entre el abanico de manifestaciones psicológicas se presentaron síntomas tales como: disminución en la concentración y propensión de olvidos de hechos recientes, malestares psicosomáticos, falta de atención y empobrecimiento en el desempeño diario.

\section{Bienestar psicológico, emocional y social}

Se considera preciso destacar la concepción de salud aportada por la Organización Mundial de la Salud (2004), donde la describe como un estado completo de bienestar físico, mental y social, y no meramente la ausencia de enfermedad. Es así como diversos investigadores pusieron el foco en el estudio de la felicidad humana desde el punto de vista de dos concepciones consideradas como opuestas: el bienestar subjetivo o hedónico y el bienestar psicológico o eudaemónico (Kahneman, 1999; Ryan \& Deci, 2001; Waterman, 1993). Desde la concepción subjetiva, Diener y Biswas-Diener (2008, 2018) pusieron de relevancia el papel de la satisfacción vital y de las emociones positivas como componentes hacia el logro de la felicidad e informaron que la longevidad, la salud física, mejores puestos laborales y una alta calidad en las relaciones interpersonales se encontraban asociadas a la felicidad. Por otro lado, el bienestar psicológico se encuentra en la realización de actividades congruentes con valores personales y que suponen un compromiso pleno, con el que las personas se sienten vivas y auténticas (Waterman, 1993). Ryff (1995) lo definió como el desarrollo del verdadero potencial de uno mismo. En consecuencia, el bienestar psicológico no sería el principal motivo en la vida del hombre, sino más bien el resultado de una vida bien vivida (Ryff \& Keyes, 1995; Ryff \& Singer, 1998).

Keyes $(2002 ; 2005 ; 2006 ; 2007)$ y Huppert y So (2011; 2013) realizaron un intento por integrar ambas tradiciones (hedónica y eudaemónica), de esta forma, buscaron dar explicación al funcionamiento mental mediante el concepto de flourishing, proponiendo que las personas pueden experimentar la sensación de bienestar subjetivo y psicológico. De este modo, Keyes et al. (2008) han estudiado la salud mental bajo la concepción de que involucra algo positivo, y no simplemente la ausencia de psicopatología.

A través de una evaluación continua y el diagnóstico categórico, Keyes $(2005 ; 2013)$ propuso un modelo de dos continuos, ubicando a las enfermedades mentales en uno de los extremos y a la salud mental en el extremo opuesto. En consecuencia, aquellas personas con presencia de florecimiento experimentarían un alto nivel 
de bienestar subjetivo y psicológico, las personas situadas en el extremo opuesto experimentarían sentimientos de vacío, falta de interés y compromiso, y se encontrarían cercanas al desarrollo de patologías mentales, tales como ansiedad, depresión, entre otras, mientras que quienes se situaran en el medio presentarían un estado de salud mental moderado. Keyes (2007) planteó que, en el polo languideciente, se encontrarían una serie de síntomas de salud mental que se encuentran incluidos en los criterios del Manual Diagnóstico y Estadístico de los Trastornos Mentales (DSM, por sus siglas en inglés).

Considerando la perspectiva hasta aquí expuesta, se podría afirmar que el logro de un estado de salud mental completo se encuentra supeditado tanto a la inexistencia de síntomas psicopatológicos como a la vivencia de bienestar global de la persona, y en sentido opuesto para el polo languideciente. Las personas con una salud mental floreciente tienen un alto rendimiento hedónico y positivo. Por el contrario, las personas con una salud mental lánguida presentan un funcionamiento hedónico bajo y se caracterizan por bajos niveles de bienestar. Se considera que las personas que no prosperan ni languidecen tienen una salud mental moderada (Keyes, 2005). Los estudios han demostrado diferencias sustanciales en la prevalencia de bienestar emocional, psicológico y social entre la población adulta de diversos países, por ejemplo, en Corea del Sur el 8 \% (Lim et al., 2013), en Canadá el $72.5 \%$ (Gilmour, 2014), en los EE. UU el 18 \% (Keyes \& Grzywacz, 2005), en China el 43 \% (Yin et al., 2013) y en Francia el 68 \% (Salama-Younes, 2011). En la mayoría de estos países las categorías de salud mental moderada y floreciente estaba compuesta por población de adultos mayores, mientras que las tasas con niveles de salud mental languideciente, en población adulta (Gilmour, 2014; Lim et al ., 2013). Respecto a la variable sexo, los estudios informaron niveles más altos de salud mental en hombres italianos y estadounidenses (Keyes, 2005; Petrillo et al., 2015) y en mujeres holandesas (Westerhof \& Keyes, 2010).

Finalmente, en Argentina, Góngora y Castro Solano (2017) descubrieron que tener cualquier trastorno mental disminuía significativamente el estado de salud mental, las personas tenían una salud mental más languideciente y menos floreciente en todas las categorías de los trastornos mentales consideradas en el estudio (ej., depresión, ansiedad, obsesión y compulsión, ansiedad fóbica, somatización). Además, informaron que la depresión fue el trastorno clínico que mostró el efecto más significativo en los niveles de salud mental languideciente, seguido del trastorno de ansiedad generalizado.

En vista de lo antes expuesto sobre la importancia que reviste la salud mental de los seres humanos y teniendo en cuenta el contexto en el que la población Argentina se encuentra, se delinearon los siguientes objetivos: 1) describir la prevalencia de las categorías de salud mental en adultos argentinos en contexto de pandemia por COVID-19; 2) comparar las categorías de salud mental en adultos argentinos en contexto de pandemia por COVID-19, según bienestar psicológico, y 3) comparar las categorías de salud mental de adultos argentinos en contexto de pandemia por COVID-19, según la capacidad para expresar sus emociones y mantener el contacto social.

\section{Método}

\section{Muestra}

La muestra estaba constituida por 888 adultos argentinos en contexto de pandemia por COVID-19. La edad promedio fue de $36.98(D E=15.37$, Mín $=$ 18, Máx = 84). El $51.2 \%(n=455)$ eran mujeres, y el $48.4 \%(n=433)$ eran varones. En cuanto a su lugar de residencia, el $39.9 \%(n=354)$ informó vivir en la provincia del Chaco, el $20.4 \%(n=181)$ en Buenos Aires, el $9.5 \%(n=84)$ en la Ciudad de Buenos Aires, el $8.1 \%$ $(n=72)$ en la provincia de Córdoba, el $6 \%(n=53)$ en la provincia de Corrientes, el $4.8 \%(n=43)$ en la provincia de San Juan, el $3 \%(n=27)$ en la provincia de Santa Fe, el $2.9 \%(n=26)$ en la provincia de Formosa, el $1.7 \%(n=15)$ en la provincia de Río Negro y el $3.6 \%(n=33)$ restante se encontraban distribuidos entre las provincias de Chubut, Jujuy, La Pampa, La Rioja, Mendoza, Misiones, Neuquén, Salta, San Luis, Santa Cruz, Santiago del Estero y Tucumán. El $53.5 \%(n=475)$ dijo estar soltero, el $22.7 \%(n=202)$ estar casado, el $12.2 \%$ $(n=108)$ estar conviviendo con su pareja, el $8.2 \%$ $(n=73)$ estar divorciado y el $3.4 \%(n=30)$ ser viudo. En cuanto al nivel de estudios máximos alcanzados, el $41.2 \%(n=366)$ informó haber concluido sus estudios universitarios, el $31.3 \%(n=278)$ terminó sus estudios 
secundarios, el $15.9 \%(n=141)$ finalizó sus estudios terciarios, el $10.6 \%(n=94)$ concluyó sus estudios superiores y el $1 \%(n=9)$ finalizó la escolaridad primaria. Finalmente, en lo que al nivel socioeconómico se refiere, el $92.2 \%(n=819)$ informó pertenecer a un estrato social medio, el $7 \%(n=62)$ bajo y el $0.8 \%(n=7)$ alto.

\section{Medición}

Encuesta Sociodemográfica. Para el presente estudio se diseñó un cuestionario, mediante el cual se indagaron datos sobre el sexo, la edad, el estado civil, el lugar de residencia de la muestra, así como el nivel de estudios y nivel socioeconómico.

Cuestionario ad hoc. Se confeccionó un cuestionario bajo los lineamientos de la Organización Mundial de la Salud (2015), mediante el cual se recolectó información sobre la necesidad de expresar emociones y estar en contacto con sus seres queridos. Las preguntas elegidas se encontraban relacionadas al interés del estudio realizado. Se seleccionaron dos preguntas cerradas con respuesta dicotómica $(s i ́$ - no). Un ejemplo de las preguntas elegidas es: "ibusca expresar sus emociones con su círculo cercano?".

Mental Health Continuum-Short Form (MHC-SF; Keyes, 2005). Es una escala compuesta de 14 ítems que evalúan el bienestar emocional (3 ítems), psicológico (6 ítems) y social (5 ítems). La escala tiene un formato de respuesta Likert con 5 opciones. Se responde con relación a cómo se ha sentido el participante en el último mes, y va de 0 (nunca) a 5 (todos los días). El MHC-SF ha demostrado tener una buena consistencia interna ( $>$ .70), y presenta una adecuada validez discriminante (estructura de tres factores, bienestar emocional, personal y social) en estudios realizados con población adulta en diferentes países (Gallagher, López \& Preacher, 2009; Gilmour, 2014; Joshanloo et al., 2013; Karas, et al., 2014; Lamers et al., 2011; Petrillo et al., 2015; Robitschek \& Keyes, 2009; Yin et al., 2013). Para este estudio se utilizó la versión argentina. Un estudio realizado en población local confirmó las propiedades psicométricas del instrumento (adecuada confiabilidad, estructura factorial y validez convergente (Lupano Perugini et al., 2016)). En la muestra del presente estudio la consistencia interna del instrumento fue de alfa de Cronbach igual a 0.86.
Escala de Bienestar Psicológico para Adultos (BIEPS-A; Casullo, 2002). Esta versión constituye una adaptación de la Escala de Bienestar Psicológico para Adolescentes BIEPS-J de la autora, que toma como referencia las propuestas teóricas de Schmutte y Ryff (1997). Consta de 13 ítems que se puntúan de 1 a 3 (de acuerdo; ni de acuerdo ni en desacuerdo; en desacuerdo). La puntuación obtenida en la escala oscila entre los 13 y los 39 puntos. Evalúa cuatro factores: aceptación/control de situaciones (3 ítems), autonomía (3 ítems), vínculos sociales (3 ítems) y proyectos (4 ítems). La consistencia interna del instrumento del estudio original era excelente y en la muestra aquí utilizada los alfas de Cronbach obtenidos fueron aceptación/control $=.70$, autonomía $=.74$, vínculos sociales $=.77$, moderación $=.79$ y proyectos $=.73$.

\section{Procedimiento}

Los datos fueron recolectados mediante un muestreo no probabilístico y voluntario en población general Argentina en contexto de pandemia por COVID-19. En primer lugar, se digitalizaron los instrumentos para su posterior distribución mediante google forms. Luego, teniendo en cuenta el contexto, el proceso de reclutamiento de datos se realizó mediante la distribución de las técnicas a través de las redes sociales Facebook, Twitter, Instagram y WhatsApp. De esta forma, se obtuvo un mayor alcance en las diferentes provincias que componen la República Argentina; este criterio se sustenta en que cada provincia incluye a diferentes municipios y localidades. El formulario contenía en su portada como campo obligatorio aceptar el consentimiento informado aprobado por el Comité de Ética en Investigación de la Asociación de Psicólogos Especializados en Catástrofes Argentina y por la Ley 25.326 de protección de los datos personales, que se ocupa de las implicancias éticas de las investigaciones en salud en las que participen seres humanos, con el fin de proteger sus derechos fundamentales, ponderando, a su vez, la necesidad de promover la investigación en salud.

\section{Análisis de datos}

Para clasificar los niveles de salud mental según el MHC-SF se utilizó el procedimiento estándar diseñado por Keyes (2005). Las personas que reportaron experimentar “todos los días" o "casi todos los días" al menos uno de 
los tres indicadores de bienestar hedónico y al menos seis de los once indicadores de funcionamiento positivo durante el pasado mes se ubicaron en la categoría de salud mental floreciente. Las personas que reportaron niveles bajos (es decir, "nunca" o "una o dos veces" durante el mes pasado) en al menos un indicador de bienestar hedónico y bajos niveles en al menos seis indicadores de funcionamiento positivo fueron diagnosticados con salud mental languideciente. El resto de los participantes que no entraban en las dos categorías anteriores se clasificaron con salud mental moderada. Se efectuó en primer lugar un cálculo de frecuencias y porcentajes para determinar la prevalencia de las categorías de salud mental, a su vez, para analizar las diferencias en los porcentajes sociodemográficos se utilizó la prueba de independencia Chi cuadrado. Posteriormente, se realizaron pruebas de análisis de varianza (MANOVA) para comparar las variables bienestar psicológico, expresión de emociones y contacto con seres queridos de acuerdo con las categorías de salud mental evaluadas por el MHC-SF. Los datos fueron sistematizados y analizados con el programa estadístico SPSS-22.0.

\section{Resultados}

A continuación se presentan los resultados obtenidos. Se exponen, en primer lugar, los cálculos de frecuencias y porcentajes, mediante los cuales se determinó la prevalencia de salud mental en adultos argentinos en contexto de pandemia por COVID-19. Posteriormente, se presenta la prueba de independencia
Chi cuadrado, con la cual se analizaron las diferencias en los porcentajes sociodemográficos según los niveles de salud mental. Por último, se informan las pruebas de análisis de varianza (MANOVA) utilizadas para comparar las variables de bienestar de acuerdo con los niveles de salud mental, y para el análisis de diferencias en la capacidad de haber expresado emociones y haber estado en contacto con los seres queridos de acuerdo al nivel de salud mental.

\section{Prevalencia de las categorías de salud mental en adultos argentinos en contexto de pandemia por COVID-19.}

Se calcularon los niveles de salud mental utilizando el MHC-SF en la muestra del presente estudio. La mayor parte de la muestra mostró una salud mental languideciente $66.7 \%(n=592)$, un porcentaje menor $23.2 \%(n=206)$ una salud mental floreciente y el $10 \%(n=89)$ restante fue clasificado como salud mental moderada.

Se realizaron pruebas de independencia Chi cuadrado para el análisis de las diferencias en los datos sociodemográficos según los niveles de salud mental. Se halló una relación significativa entre la variable sexo y los niveles de salud mental, $X^{2}(1, n=887)=9.14, p<.01$ (ver Tabla 1). Entre los hombres $(71.1 \%, n=308)$ hubo una proporción mayor de sujetos con niveles de salud mental languideciente que entre las mujeres $(62.6 \%, n$ $=284)$, y entre las mujeres hubo mayores participantes con niveles de salud mental moderado $(10.1 \%, n=46)$ y floreciente $(27.3 \%, n=124)$ que entre los hombres $(9.9 \%, n=43 ; 18.9 \%, n=82)$.

\section{Tabla 1}

Prevalencia de salud mental según estado civil $(N=888)$

\begin{tabular}{|c|c|c|c|c|c|c|c|c|c|c|}
\hline \multicolumn{11}{|c|}{$X^{2}(2)=17.286 ; p=.020$} \\
\hline & \multicolumn{2}{|c|}{ Soltero } & \multicolumn{2}{|c|}{ Casado } & \multicolumn{2}{|c|}{ Concubinato } & \multicolumn{2}{|c|}{ Divorciado } & \multicolumn{2}{|c|}{ Viudo } \\
\hline & $\mathbf{n}$ & $\%$ & $\mathbf{n}$ & $\%$ & $\mathbf{n}$ & $\%$ & $\mathbf{N}$ & $\%$ & $\mathbf{n}$ & $\%$ \\
\hline Floreciente & 124 & 26.1 & 31 & 15.4 & 27 & 25.0 & 19 & 26.0 & 5 & 16.7 \\
\hline Moderado & 56 & 11.8 & 14 & 7.0 & 10 & 9.3 & 7 & 9.6 & 2 & 6.7 \\
\hline Languideciente & 295 & 62.1 & 156 & 77.6 & 71 & 65.7 & 46 & 64.4 & 23 & 76.7 \\
\hline
\end{tabular}

Además, se halló una relación significativa entre la variable estado civil y los niveles de salud mental, $X^{2}$
$(1, n=887)=17.28, p<.02($ ver Tabla 2$)$. Entre las personas casadas hubo una proporción mayor de sujetos 
que reportaron un nivel de salud mental languideciente que entre las demás categorías de estado civil, y entre las personas que informaron estar solteras presentaron mayores niveles de salud mental moderado y floreciente que entre las demás categorías. Sin embargo, no se hallaron relaciones significativas entre las variables nivel educativo $(p>30)$, clase social $(p>30)$ y niveles de salud mental.

\section{Tabla 2}

Prevalencia de salud mental según sexo $(n=888)$

\begin{tabular}{lcccc}
\hline \multicolumn{4}{c}{$\mathbf{X}^{\mathbf{2}}(\mathbf{2})=\mathbf{9 . 1 4 5} ; \mathbf{p}=\mathbf{. 0 1 0}$} \\
\multicolumn{2}{c}{ Femenino } & \multicolumn{2}{c}{ Masculino } \\
\hline Floreciente & $\mathbf{n}$ & $\mathbf{\%}$ & $\mathbf{N}$ & $\mathbf{\%}$ \\
Moderado & 46 & 27.3 & 82 & 18.9 \\
Languideciente & 284 & 10.1 & 43 & 9.9 \\
\hline \hline
\end{tabular}

\section{Comparación del bienestar psicológico de} acuerdo con los niveles de salud mental

Se procedió a realizar un análisis multivariado de varianza con prueba post hoc de Bonferroni debido a que el análisis de homogeneidad de varianzas de Levene demostró que estas eran iguales entre los grupos (ver Tabla 3). Los resultados del análisis mostraron que los individuos con salud mental languideciente presentaban niveles significativamente menores en las dimensiones de aceptación-control $\left(p<.000 ; \eta^{2}=.03\right)$, autonomía $(p<.001 ;$ $\left.\eta^{2}=.02\right)$, proyectos $\left(p<.013 ; \eta^{2}=.01\right)$ y en el total de bienestar psicológico $\left(p<.000 ; \eta^{2}=.03\right)$ que los individuos con salud mental moderada y floreciente. Sin embargo, no se hallaron diferencias significativas entre los grupos para la dimensión vínculos $(p>.06$; $\left.\eta^{2}=.00\right)$. Las diferencias entre los niveles de salud mental y las dimensiones de bienestar psicológicos fueron entre moderadas y bajas (Ellis, 2010).

\section{Tabla 3}

Análisis de la varianza del bienestar psicológico de acuerdo con los niveles de salud mental

\begin{tabular}{|c|c|c|c|c|c|c|c|c|}
\hline \multirow[t]{2}{*}{ Escala } & \multicolumn{2}{|c|}{$\begin{array}{l}\text { Salud Mental } \\
\text { Floreciente }\end{array}$} & \multicolumn{2}{|c|}{$\begin{array}{c}\text { Salud Mental } \\
\text { Moderada }\end{array}$} & \multicolumn{2}{|c|}{$\begin{array}{c}\text { Salud Mental } \\
\text { Languideciente }\end{array}$} & \multirow[b]{2}{*}{$F(2,886)$} & \multirow[b]{2}{*}{$\eta^{2}$} \\
\hline & $M$ & $D E$ & $M$ & $D E$ & $M$ & $D E$ & & \\
\hline Aceptación-Control & 1.44 & 0.43 & 1.33 & 0.38 & 1.27 & 0.38 & $14.04 * * *$ & .031 \\
\hline Autonomía & 1.76 & 0.54 & 1.67 & 0.49 & 1.59 & 0.54 & $7.33 * *$ & .020 \\
\hline Vínculos & 1.28 & 0.34 & 1.25 & 0.31 & 1.25 & 0.33 & $0.52 n s$ & .001 \\
\hline Proyectos & 1.35 & 0.35 & 1.23 & 0.26 & 1.28 & 0.38 & $4.37 *$ & .010 \\
\hline BIEPS-A Total & 1.45 & 0.26 & 1.36 & 0.24 & 1.35 & 0.30 & $10.43 * * *$ & .030 \\
\hline
\end{tabular}

Nota: $* * * \mathrm{p}<.000 ; * * \mathrm{p}<.001 ; * \mathrm{p}<.013$

\section{Capacidad para expresar emociones y estar en contacto con seres queridos de acuerdo al nivel de salud mental}

Frente a la pregunta sobre si expresaban sus emociones con su círculo cercano, el $35.2 \%(n=313)$ informó que lo realizaba pocas veces, el $29.5 \%(n=$ 262) muchas veces, el $26.7 \%(n=237)$ casi siempre y el $8.6 \%(n=76)$ restante respondió casi nunca. Se procedió a realizar un análisis multivariado de varianza (MANOVA) con análisis post hoc de Bonferroni para comparar la expresión de las emociones y el contacto con los seres queridos según el nivel de salud mental. Este procedimiento se seleccionó porque el análisis de homogeneidad de varianzas de Levene indicó que estas eran iguales entre los grupos. 
Los resultados del análisis mostraron que los individuos que respondieron que casi siempre expresaban sus emociones presentaron niveles significativamente mayores de bienestar emocional $\left(p=.000 ; \eta^{2}=.091\right)$, social $\left(p=.000 ; \eta^{2}=.068\right)$, psicológico $\left(p=.000 ; \eta^{2}=.11\right)$ y total $(p=.000$; $\eta^{2}=.12$ ) en comparación con quienes respondieron haber expresado sus emociones muchas veces, pocas veces o casi nunca. Las diferencias entre los niveles de salud mental y la capacidad para expresar emociones presentó magnitudes del tamaño del efecto entre moderadas y altas (Ellis, 2010; ver Tabla 4).

\section{Tabla 4}

Análisis de la varianza del bienestar psicológico de acuerdo con la expresión de emociones

\begin{tabular}{lccccccccccc}
\hline \hline \multirow{2}{*}{ Escala } & \multicolumn{2}{c}{ Casi Nunca } & \multicolumn{2}{c}{ Pocas Veces } & \multicolumn{1}{c}{ Muchas Veces } & \multicolumn{2}{c}{ Casi Siempre } & & \\
\cline { 2 - 8 } & $\boldsymbol{M}$ & $\boldsymbol{D E}$ & $\boldsymbol{M}$ & $\boldsymbol{D E}$ & $\boldsymbol{M}$ & $\boldsymbol{D E}$ & $\boldsymbol{M}$ & $\boldsymbol{D E}$ & $\boldsymbol{F}(\mathbf{3 , 8 8 2})$ & $\boldsymbol{\eta}^{\mathbf{2}}$ \\
\hline $\begin{array}{l}\text { Bienestar } \\
\text { Emocional }\end{array}$ & 2.60 & 1.29 & 3.01 & 1.09 & 3.22 & 1.16 & 3.73 & 0.92 & $29.46^{*}$ & .091 \\
$\begin{array}{l}\text { Bienestar Social } \\
\text { Bienestar }\end{array}$ & 1.40 & 0.89 & 1.63 & 0.99 & 1.81 & 1.06 & 2.25 & 1.07 & $21.60 *$ & .068 \\
$\begin{array}{l}\text { Psicológico } \\
\text { Bienestar Total }\end{array}$ & 2.86 & 1.30 & 2.91 & 1.10 & 3.28 & 1.05 & 3.83 & 0.81 & $39.33 *$ & .118 \\
\hline \hline
\end{tabular}

Nota: $* \mathrm{p}<.000$

Al momento de indagar sobre si mantenían contacto con sus seres queridos, así sea de forma virtual, el $43 \%(n=382)$ respondió que lo realizaba casi siempre, el $37.7 \%(n=335)$ muchas veces, el $16 \%$ $(n=142)$ pocas veces y el $3.3 \%(n=29)$ restante refirió que casi nunca lo realizaba. Con respecto al análisis de las diferencias de los distintos tipos de bienestar según las personas hayan establecido contacto con sus seres queridos al menos de forma virtual, los resultados informaron que las personas que casi siempre mantuvieron el contacto presentaron niveles significativamente mayores de bienestar emocional $\left(p=.000 ; \eta^{2}=.06\right)$, social $\left(p=.000 ; \eta^{2}=.06\right)$, psicológico $\left(p=.000 ; \eta^{2}=.08\right)$ y total $(p=.000$; $\eta^{2}=.09$ ) en comparación con quienes respondieron muchas veces, pocas veces o casi nunca. Aquí también se pudo observar que las diferencias entre los niveles de salud mental y la capacidad para expresar emociones presentó magnitudes del tamaño del efecto entre moderadas y altas (Ellis, 2010) (Tabla 5).

Tabla 5

Análisis de la varianza del bienestar psicológico según contacto con seres queridos

\begin{tabular}{|c|c|c|c|c|c|c|c|c|c|c|}
\hline \multirow{2}{*}{ Escala } & \multicolumn{2}{|c|}{ Casi Nunca } & \multicolumn{2}{|c|}{ Pocas Veces } & \multicolumn{2}{|c|}{ Muchas Veces } & \multicolumn{2}{|c|}{ Casi Siempre } & \multirow[b]{2}{*}{$F(3,882)$} & \multirow[b]{2}{*}{$\eta^{2}$} \\
\hline & $M$ & $D E$ & $M$ & $D E$ & $M$ & $D E$ & $M$ & $D E$ & & \\
\hline $\begin{array}{l}\text { Bienestar } \\
\text { Emocional }\end{array}$ & 2.22 & 1.19 & 2.80 & 1.15 & 3.30 & 1.09 & 3.41 & 1.10 & $18.85 *$ & .060 \\
\hline Bienestar Social & 1.22 & 0.91 & 1.34 & 0.93 & 1.89 & 1.07 & 2.01 & 1.05 & $17.81 *$ & .060 \\
\hline $\begin{array}{l}\text { Bienestar } \\
\text { Psicológico }\end{array}$ & 2.11 & 1.27 & 2.85 & 1.17 & 3.25 & 1.03 & 3.51 & 1.02 & $25.57 *$ & .080 \\
\hline Bienestar Total & 1.82 & 0.95 & 2.30 & 0.91 & 2.78 & 0.90 & 2.95 & 0.94 & $28.69 *$ & .090 \\
\hline
\end{tabular}

Nota: $* \mathrm{p}<.000$ 


\section{Discusión}

El primer objetivo de este estudio fue describir la prevalencia de las categorías de salud mental en adultos argentinos en contexto de pandemia por COVID-19. Los resultados mostraron que entre los hombres hubo una proporción mayor de sujetos con niveles de salud mental languideciente que entre las mujeres. En las mujeres hubo mayores participantes con niveles de salud mental moderado y floreciente que entre los hombres. Este hallazgo se encuentra en contraposición a estudios internacionales precedentes, donde hombres italianos y estadounidenses reportaron mayores niveles de salud mental floreciente en contraposición a las mujeres (Keyes, 2005; Petrillo et al., 2015), y en estudios con población canadiense y coreana, donde no encontraron diferencias significativas según la variable sexo para los niveles de salud mental (Gilmour, 2014; Góngora \& Castro Solano, 2017; Lim et al., 2013; Yin et al., 2013). Probablemente, estas diferencias se encuentren no solo en relación al contexto de pandemia que se encuentra atravesando el mundo, sino también en las formas de afrontar situaciones complejas. En este sentido, diferentes estudios demostraron que el afrontamiento de las mujeres es funcional y con características relacionadas a la resiliencia (Forés \& Granés, 2008; Rutter, 1999; Manciaux et al., 2003). Es importante subrayar que el bienestar psicológico está determinado socioculturalmente, existiendo una interrelación dialéctica entre lo biológico, lo social y lo psicológico (Fernández Ríos, 2003); por lo cual, se podría inferir que estos resultados se encuentren relacionados a los componentes culturales de la muestra analizada.

Además, este estudio reveló que entre las personas casadas hubo una proporción mayor de sujetos que reportaron un nivel de salud mental languideciente que entre las demás categorías de estado civil, y que entre las personas que informaron estar solteras presentaron mayores niveles de salud mental moderada y floreciente que entre las demás categorías de estado civil. Este resultado se encuentra en consonancia con lo informado por Zimmermann y Easterlin (2006), quienes refirieron que el estado civil se encontraría relacionado al bienestar psicológico individual más que con el bienestar de la pareja; motivo por el cual se podría pensar que aquellas personas que se encuentran en una relación de pareja experimentarían sentimientos de bienestar y funcionamiento efectivo independientemente del vínculo. Es importante que en futuros estudios se puedan evaluar el nivel de bienestar teniendo en cuenta la interacción entre sexo, estado civil y ocupación.

El segundo objetivo de este trabajo se refirió a comparar las categorías de salud mental en adultos argentinos en contexto de pandemia por COVID-19 según bienestar psicológico. Los resultados obtenidos informaron que los individuos con salud mental languideciente presentaban niveles significativamente menores en las dimensiones de aceptación-control, autonomía, proyectos $\mathrm{y}$, en el total, de bienestar psicológico que los individuos con salud mental moderada y floreciente. Este resultado se encuentra en consonancia con los estudios sobre salud mental de población en contexto de pandemia por COVID-19 en otros países (Brooks et al., 2020; Chen et al., 2020; Hoof, 2020). El presente hallazgo indica que los adultos argentinos en contexto de pandemia por COVID-29 presentan un funcionamiento hedónico bajo, es decir, languideciente; en términos de Keyes (2005, 2013), representa la ausencia de salud mental caracterizada por desilusiones, sentimientos relacionados a que la vida carece de significado, lo que provoca inestabilidad emocional. Es así como se encuentran limitados ante la posibilidad de establecer propósitos en la vida. Futuros estudios podrían centrarse en la influencia de los síntomas psicopatológicos sobre el bienestar psicológico en el contexto actual de pandemia.

El tercer objetivo versaba en comparar las categorías de salud mental de adultos argentinos en contexto de pandemia por COVID-19, según la capacidad para expresar sus emociones y mantener el contacto social. En consonancia con diversos estudios relacionados a los beneficios en expresar emociones y mantener el contacto con seres queridos (Avsec etal., 2016; Peterson \& Seligman, 2005; Seligman, 2011), los resultados informaron que los individuos al responder que casi siempre expresaban sus emociones y que mantenían el contacto con sus seres queridos, presentaron niveles significativamente mayores de bienestar emocional, social y psicológico, en comparación con los que respondieron muchas veces, pocas veces o casi nunca. Este hallazgo indica que las personas con una salud mental floreciente tienen un alto 
rendimiento hedónico y positivo. Se podría concluir que los sujetos de población argentina, al estar en contacto con sus seres queridos y expresar sus emociones, experimentan emociones positivas, satisfacción con la vida, sensación de crecimiento personal, integración y contribución social.

Dentro de las limitaciones del presente estudio se pueden mencionar la dificultad relacionada a la muestra, debido a que la recolección de datos se realizó con procedimientos de selección no probabilísticos, lo cual influyó directamente en los supuestos que subyacen a la mayoría de los análisis estadísticos; asimismo, el carácter transversal que no permite evaluar el valor predictivo, y, finalmente, cabe mencionar que, debido al carácter de novedad que reviste el estudio del bienestar, en contexto de pandemia no fue factible encontrar estudios previos que hayan utilizado el modelo y las variables aquí analizadas.

A modo de conclusión general, este estudio ha podido arrojar modestas evidencias sobre la influencia negativa que generaría la distancia social y la falta de capacidad para expresar emociones en la salud mental de los adultos argentinos en contexto de pandemia por COVID-19. A su vez, se considera de suma relevancia la atención de estos aspectos para el desarrollo de intervenciones dirigidas hacia el bienestar psicológico, emocional y social, conceptualizados como un estado completo de la salud mental.

\section{Referencias}

Avsec, A., Kavčič, T., \& Jarden, A. (2016). Synergistic paths to happiness: Findings from seven countries. Journal of Happiness Studies, 17(4), 13711390. Available at https://cutt.ly/QfMdVa9

Brooks, S. K., Webster, R. K., Smith, L. E., Woodland, L., Wessely, S., Greenberg, N., \& Rubin, G. J. (2020). Rapid Review. Lancet, 395, 912-20. https:// doi.org/10.1016/S0140-6736(20)30460-8

Chen, N., Zhou, M., Dong, X., Qu, J., Gong, F., Han, Y., ... \& Yu, T. (2020). Epidemiological and clinical characteristics of 99 cases of 2019 novel coronavirus pneumonia in Wuhan, China: a descriptive study. The Lancet, 395(10223), 507-513. https:// doi.org/10.1016/S0140-6736(20)30211-7
Diener, E., \& Biswas-Diener, R. (2008). Rethinking happiness: The science of psychological wealth. Malden: Blackwell Publishing.

Ellis, P. D. (2010). The essential guide to effect sizes: Statistical power, meta-analysis, and the interpretation of research results. Cambridge University Press.

Fernández-Ríos, L., \& Comes, J. M. (2009). Una revisión crítica de la historia y situación actual de la psicología positiva. Anuario de Psicología Clínica y de la Salud, 5, 7-13. Available at https://cutt.ly/ $\mathrm{CgqWeRv}$

Forés, A., \& Granés, J. (2008). La resiliencia. Crecer desde la adversidad. Barcelona: Plataforma Editorial

Gallagher, M. W., Lopez, S. J., \& Preacher, K. J. (2009). The hierarchical structure of well-being. Journal of personality, 77(4), 1025-1050. https://doi. org/10.1111/j.1467-6494.2009.00573.x

Gilmour, H. (2014). Positive Mental Health and Mental Illness. Health Reports, 25, 3-9.

Góngora, V. C., \& Solano, A. C. (2017). Assessment of the Mental Health Continuum in a sample of Argentinean Adults. Psychology, 8(3), 303-318. https://doi.org/10.4236/psych.2017.83018

Guiroy, A., Gagliardi, M., Coombes, N., Landriel, F., Zanardi, C., Camino Willhuber, G., ... \& Valacco, M. (2020). COVID-19 Impact Among Spine Surgeons in Latin America. Global Spine Journal. https://doi.org/10.1177/2192568220928032

Hale, T., Webster, S., Petherick, A., Phillips, T., \& Kira, B. (2020) Oxford COVID-19 government response tracker. Blavatnik School of Government, 25.

Huppert, F. A., \& So, T. T. C. (2013). Flourishing Across Europe: Application of a New Conceptual Framework for Defining Well-Being. Social Indicators Research, 110(3), 837-861. http://dx.doi. org/10.1007/s11205-012-0030-z.

Jeong, H., Woo, H., Song, Y. J., Ki, M., Min, J., Cho, J., \& Chae, J. H. (2016). Mental health status of people isolated due to Middle East Respiratory Syndrome. Epidemiology and Health, 38: e2016048. https://doi.org/10.4178/epih.e2016048

Joshanloo, M., Wissing, M. P., Khumalo, I. P., \& Lamers, S. M. (2013). Measurement invariance of the Mental Health Continuum-Short Form (MHCSF) across three cultural groups. Personality and 
Individual Differences, 55(7), 755-759. https://doi. org/10.1016/j.paid.2013.06.002

Kahneman, D. (1999). Objective happiness. Well-being: The foundations of hedonic psychology, 3(25), 1-23. https://psycnet.apa.org/record/1999-02842-001

Karaś, D., Cieciuch, J., \& Keyes, C. L. (2014). The polish adaptation of the mental health continuumshort form (MHC-SF). Personality and Individual Differences, 69, 104-109. https://doi.org/10.1007/ s11205-014-0629-3

Keyes, C. L. (2002). The mental health continuum: From languishing to flourishing in life. Journal of Health and Social Behavior, 207-222. Available at http:// www.jstor.org/stable/3090197

Keys, C. L. (2005). Mental Illness and/or Mental Health? Investigating Axioms of the Complete State Model of Health. Journal of Consulting and Clinical Psychology, 3, 539-548. https://doi.org/10.1037/0022006X.73.3.539

Keyes, C. L. (2006). Subjective well-being in mental health and human development research worldwide: An introduction. Social Indicators Research, 77(1), 1-10. https://doi.org/10.1007/s11205014-0668-9

Keye, C. L. (2007). Promoting and protecting mental health as flourishing: A complementary strategy for improving national mental health. American Psychologist, 62(2), 95. https://doi.org/10.1037/0003066X.62.2.95

Keyes, C. L. M., \& Grzywacz, J. G. (2005). Health as a complete state: the added value in work performance and healthcare costs. Journal of Occupational and Environmental Medicine, 47, 523-532. https:// doi.org.10.1097/01.jom.0000161737.21198.3a

Keyes, C. L., Wissing, M., Potgieter, J. P., Temane, M., Kruger, A., \& Van Rooy, S. (2008). Evaluation of the mental health continuum-short form (MHCSF) in setswana-speaking South Africans. Clinical Psychology \& Psychotherapy, 15(3), 181-192. https:// doi.org/10.1002/cpp.572

Lamers, S. M., Westerhof, G. J., Bohlmeijer, E. T., ten Klooster, P. M., \& Keyes, C. L. (2011). Evaluating the psychometric properties of the mental health continuum-short form (MHC-SF). Journal of Clinical Psychology, 67(1), 99-110. https://doi. org/10.1002/jclp.20741

Lee, A.M., Wong, J., McAlonan, G. M., Cheung, V., Cheung, C. C., Sham, P., Chu, C., Wong, P., Tsang, K., \& Chua, S. A., (2007). Stress and Psychological Distress Among SARS Survivors 1 Year After the Outbreak. The Canadian Journal of Psychiatry, 52(4), 233-240.

Lim, Y.-J., Ko, Y.-G., Shin, H.-C., \& Cho, Y. (2013). Prevalence and Correlates of Complete Mental Health in the South Korean Adult Population. In C. L. M. Keyes (Ed.), Mental Well-Being (pp. 91-109). Berlin: Springer. https://doi.org/10.1007/97894-007-5195-8_5

Manciaux, M., Vanistendael, S., Lecomte, J., \& Cyrulnik, B. (2003). La resiliencia: estado de la cuestión. En M. Manciaux (dir.), La resiliencia: resistir y rehacersex (pp. 17-27). Madrid: Gedisa. (Orig. 2001).

Morno-Proaño, G. (2020). Pensamientos distorsionados y ansiedad generalizada en COVID-19. CienciAmérica, 9(2), 251-255. http://dx.doi.org/10.33210/ ca.v9i2.314

OMS. (2020). Coronavirus. Recuperado de https://www. who.int/health-topics/coronavirus

Organización Mundial de la Salud (2004). World Health Organization Constitution. Geneva, Switzerland: Author.

Organización Panamericana de la Salud. Actualización Epidemiológica: Nuevo coronavirus (COVID-19) 14 de febrero de 2020. Washington D. C.: Organización Panamericana de la Salud; 2020.

Perugini, M. L. L., de la Iglesia, G., Solano, A. C., \& Keyes, C. L. M. (2017). The mental health continuum-short form (MHC-SF) in the Argentinean context: Confirmatory factor analysis and measurement invariance. Europe's Journal of Psychology, 13(1), 93. https://doi.org/10.5964/ejop. v13i1.1163

Peterson, Ch., Park, N., \& Seligman, M. E. P. (2005). Orientations to happiness and life satisfaction: the full life versus the empty life. Journal of Happiness Studies, 6, 25-41. https://doi.org/10.1007/ s10902-004-1278-z

Petrillo, G., Capone, V., Caso, D., \& Keyes, C. L. (2015). The Mental Health Continuum-Short Form 
(MHC-SF) as a measure of well-being in the Italian context. Social Indicators Research, $121(1)$, 291-312. https://doi.org/10.1007/s11205-0140629-3

Robitschek, C., \& Keyes, C. L. (2009). Keyes's model of mental health with personal growth initiative as a parsimonious predictor. Journal of Counseling Psychology, 56(2), 321. https://doi.org/10.1037/ a0013954

Rutter, M. (1999). Resilience concepts and findings: implications for family therapy. Journal of Family Therapy, 21, 119-144. https://doi.org/10.1111/14676427.00108

Ryan, R. M., \& Deci, E. L. (2001). On happiness and human potentials: A review of research on hedonic and eudaimonic well-being. Annual Review of Psychology, 52(1), 141-166. https://doi.org/10.1146/annurev.psych.52.1.141

Ryff, C. D. (1995). Psychological well-being in adult life. Current Directions in Psychological Science, 4(4), 99-104. https://doi.org/10.1111/1467-8721. ep10772395

Ryff, C. D., \& Keyes, C. L. M. (1995). The structure of psychological well-being revisited. Journal of Personality and Social Psychology, 69(4), 719. https://doi. org/10.1037/0022-3514.69.4.719

Ryff, C. D., \& Singer, B. (1998). The contours of positive human health. Psychological Inquiry, 9, 1-28. https://doi.org/10.1207/s15327965pli0901_1

Salama-Younes, M. (2011). Positive Mental Health, Subjective Vitality and Satisfaction with Life for French Physical Education Students. World Journal of Sport Sciences, 4, 90-97. https://cutt.ly/sfMk7bC

Schmutte, P. S., \& Ryff, C. D. (1997). Personality and well-being: reexamining methods and meanings. Journal of Personality and Social Psychology, 73(3), 549. https://doi.org/10.1037/0022-3514.73.3.549

Seligman, M. E. P. (2011). Flourish: A Visionary New Understanding of Happiness and wellbeing. New York: Free Press.
Wang, J., Wang, J. X., \& Yang, G. S. (2020). The Psychological Impact of COVID-19 on Chinese Individuals. Yonsei Medical Journal, 61(5), 438. https:// doi.org/10.3349/ymj.2020.61.5.438

Waterman, A. S. (1993). Two conceptions of happiness: Contrasts of personal expressiveness (eudaimonia) and hedonic enjoyment. Journal of Personality and Social Psychology, 64(4), 678. https://doi. org/10.1037/0022-3514.64.4.678

Westerhof, G., \& Keyes, C. L. M. (2010). Mental Illness and Mental Health: The Two Continua Model across the Lifespan. Journal of Adult Development, 17, 110-119. https://doi.org/10.1007/s10804-009-9082-y

World Health Organization. (2015). MhGAP humanitarian intervention guide (mhGAP-HIG): clinical management of mental neurological and substance use conditions in humanitarian emergencies. World Health Organization.

Yao, H., Chen, J. H., \& Xu, Y. F. (2020). Patients with mental health disorders in the COVID-19 epidemic. The Lancet Psychiatry, 7(4), e21. https://doi. org/10.1016/S2215-0366(20)30090-0

Yin, K., He, J., \& Fu, Y. (2013). Positive Mental Health: Measurement, Prevalence, and Correlates in a Chinese Cultural Context. In C. L. M. Keyes (Ed.), Mental Well-Being (pp. 111-132). Berlin: Springer. https://doi.org/10.1007/978-94-007-5195-8_6

Zaki, A. M., van Boheemen, S., Bestebroer, T. M., Osterhaus, A. D., \& Fouchier, R. A. (2012). Isolation of a novel coronavirus from a man with pneumonia in Saudi Arabia. The New England Journal of Medicine, 367, 1814-1820. https:// 10.1056/NEJMoa1211721

Zimmermann, A. C., \& Easterlin, R. E. (2006). Happily, ever after? Cohabitation, marriage, divorce, and happiness in Germany. Population and Development Review, 32, 511-528. https://www.jstor.org/stable/20058902 